\title{
Experimental Analysis of Gas-Liquid-Solid Three-Phase Flows in Horizontal Pipelines
}

\author{
Paolo Sassi $^{1}$ D $\cdot$ Youssef Stiriba ${ }^{1} \cdot$ Julia Lobera ${ }^{2} \cdot$ Virginia Palero $^{2} \cdot$ Jordi Pallarès $^{1}$
}

Received: 17 October 2019 / Accepted: 13 April 2020 / Published online: 9 May 2020

(c) The Author(s) 2020

\begin{abstract}
The dynamics of three-phase flows involves phenomena of high complexity whose characterization is of great interest for different sectors of the worldwide industry. In order to move forward in the fundamental knowledge of the behavior of three-phase flows, new experimental data has been obtained in a facility specially designed for flow visualization and for measuring key parameters. These are (1) the flow regime, (2) the superficial velocities or rates of the individual phases; and (3) the frictional pressure loss. Flow visualization and pressure measurements are performed for two and three-phase flows in horizontal $30 \mathrm{~mm}$ inner diameter and $4.5 \mathrm{~m}$ long transparent acrylic pipes. A total of 134 flow conditions are analyzed and presented, including plug and slug flows in air-water two-phase flows and air-water-polypropylene (pellets) three-phase flows. For two-phase flows the transition from plug to slug flow agrees with the flow regime maps available in the literature. However, for three phase flows, a progressive displacement towards higher gas superficial velocities is found as the solid concentration is increased. The performance of a modified Lockhart-Martinelli correlation is tested for predicting frictional pressure gradient of three-phase flows with solid particles less dense than the liquid.
\end{abstract}

Keywords Three-phase flows · Horizontal pipelines $\cdot$ Plug-to-slug flow transition · Frictional pressure drop $\cdot$ Flow visualization

\section{List of Symbols}

\section{Variables}

$D \quad$ Internal diameter of pipeline (m)

$f \quad$ Darcy-Weisbach friction factor

$g \quad$ Acceleration of gravity $\left(9.81 \mathrm{~m} / \mathrm{s}^{2}\right)$

$j \quad$ Superficial velocity $(\mathrm{m} / \mathrm{s})$

Re Reynolds number $(R e=\mathrm{v} \mathrm{D} / \nu)$

$X \quad$ Lockhart-Martinelli parameter

Paolo Sassi

paolo.sassi@urv.cat

1 Universitat Rovira i Virgili, Tarragona, Spain

2 Instituto de Investigación en Ingeniería de Aragón (I3A), Universidad de Zaragoza, Zaragoza, Spain 
$P \quad$ Pressure $(\mathrm{Pa})$

$z \quad$ Axial coordinate of the pipeline (m)

$C \quad$ Auxiliar parameter for correlations

$C_{s} \quad$ Solid concentration (volume fraction)

$S \quad$ Wetted perimeter (m)

A Cross sectional area $\left(\mathrm{m}^{2}\right)$

\section{Greek Symbols}

$\alpha \quad$ Void fraction

$\delta \quad$ Derivative

$\mu \quad$ Dynamic viscosity (Pa s)

$\Phi \quad$ LM multi-phase multiplier

$\tau \quad$ Shear stress $(\mathrm{Pa})$

$\rho \quad$ Density $\left(\mathrm{kg} / \mathrm{m}^{3}\right)$

$v \quad$ Kinematic viscosity $\left(\mathrm{m}^{2} / \mathrm{s}\right)$

\section{Subscripts}

$\begin{array}{ll}\text { atm } & \text { Atmospheric conditions } \\ f & \text { Liquid phase } \\ s & \text { Solid phase } \\ g & \text { Gas phase } \\ w & \text { Pipe wall }\end{array}$

\section{Abbreviations}

FPG Frictional pressure gradient

$G / L \quad$ Gas-liquid mixture

G/L/S Gas-liquid-solid mixture

ID Inner diameter

LM Lockhart-Martinelli parameter

$L / S \quad$ Liquid-solid mixture

$V F D \quad$ Variable frequency drive

\section{Introduction}

The dynamics of three-phase flows, gas-liquid-solid (G/L/S) mixtures, involve high complexity phenomena, whose characterization is crucial for different sectors of the industry. Three-phase flows are present in a wide range of industrial processes, from the production stages of oil and gas (Bello et al. 2005) to the different phases of refinement and production of petroleum products, and it is also involved, among others, in the transport of biomass (Miao et al. 2013) or in some chemical reactors (Scott and Rao 1971), nuclear waste decommissioning (Mao et al. 1997), pulp and paper production, and in many applications of air injections (Orell 2007). Thus it is important to optimize three phase flows, a process in which there is still plenty of room for improvement.

As highlighted by Rahman et al. (2013), there are three main parameters to consider when designing a pipeline to transport three-phase flow mixtures. (1) The first one, which is also important in single phase flows, is the frictional pressure gradient (FPG) along the installation, which allows to make predictions of power consumption during operation; (2) 
the flow regime, that is the spatial distribution of the phases over the pipe volume, which is important to establish precisely, because specific regimes may be needed for different processes; and (3) the deposition velocity which is the minimum operational velocity required to avoid accumulation of particles at the bottom of the pipeline. One of the reasons for the lack of comprehensive studies regarding G/L/S mixtures is the fact that not all parameters or required measurements are reported in the available literature.

This work aims to bridge this gap by analyzing three-phase flows inside horizontal pipelines using an experimental rig specifically designed for this type of flows. The experimental facility is fully described in Sassi et al. (2020), the pipelines have circular cross-section and are made of transparent plexi-glass for flow visualization. It has the capability to regulate and measure the inlet velocities in order to analyze every flow regime, and to measure the pressure at several sections along the pipeline. As the solid particles used in this experimentation are pellets of polypropylene, which are less dense $\left(\rho_{s}=866 \mathrm{~kg} / \mathrm{m}^{3}\right)$ than water (liquid phase), no deposition velocity is experienced.

One of the seminal works regarding multiphase flows was carried out by Lockhart and Martinelli (1949). They developed a correlation to calculate the FPG of liquid-gas mixtures in horizontal pipelines. The main idea was to determine the FPG of the mixture by calculating it as a single phase, as if each phase was flowing separately in the pipeline, and then correcting these values by the Lockhart-Martinelli (L-M) parameter. This parameter might adopt four different values regarding the flow mechanism of the phases, as it quantifies to what extent the liquid or gas flows are turbulent or dominated by viscosity. The L-M correlation has been used both in the industry and in many research works, mainly because of its simplicity and its ability to predict reliably the value of the pressure drop. Since their study, several authors have validated the correlation (Dukler et al. 1964; Mao et al. 1997; Abduvayt et al. 2003). In particular Beggs and Brill (1973) proposed a more accurate correlation for pipelines with any inclination angle. One of the goals of this work was to provide a more updated system, more convenient for some already available technological methodologies.

Regarding liquid-solid mixtures, there is a great deal of experimentation on slurry flows, because this type of flows are very frequent in the chemical industry, including manufacturing of pharmaceuticals, nano-fabrication, and oil refining among others. It is also applicable to long distance transport of materials like coal or waste tailings. In many cases hydrodynamic transport of solids can be both, more energy-efficient and have lower operating and maintenance costs than other handling methods. Most of the insights in this area came from (Gillies et al. 1997; Gillies and Shook 2000; Gillies et al. 2004; Wilson and Sellgren 2003) which classified the slurries in two types: (1) homogeneous or nonsettling slurries, and (2) heterogeneous or settling slurries. For the scope of the present research, the focus is set on heterogeneous slurries which usually exhibit approximately a Newtonian behaviour, and so the ratio between the viscosity of the slurry and the viscosity of the fluid can be considered a function of particle shape and solids concentration. Gillies et al. (2004) proposed the "SRC Two-Layer model", where the velocity and concentration distributions are approximated by step functions. This is because the particles are assumed to be concentrated mainly in the lower layer with the maximum concentration, while in the upper layer particles are uniformly distributed and suspended by turbulence, with a lower concentration.

For the study of three-phase flows, gas-liquid-solid (G/L/S) mixtures, some authors modified gas-liquid correlations to approximate the three-phase flow complexity by analysing it as a "gas-slurry" two phase flow, where the slurry phase is previously corrected by a liquid-solid two phase correlation. Several works modified the L-M correlation to be applicable to G/L/S 
mixtures by adjusting the liquid properties (Scott and Rao 1971; Kago et al. 1986; Hatate et al. 1986). Accurate predictions were obtained in Rahman et al. (2013) with an implementation of an improved L-M correlation, where the "slurry phase" pressure gradient was calculated using the "SRC Two-Layer Model".

Although there are already some significant developments in G/L/S mixture flows, to our knowledge, none of the previous works consider solids less dense than the liquid phase. There are some few exceptions regarding ice slurry flows (Edelin et al. 2015; Stutz et al. 2000) in which the authors analyzed two-phase, L/S mixtures, flows where the ice was modeled using polypropylene in order to compute the FPG and analyze the transport efficiency. In both works, the authors concluded that the particle size had little impact in the pressure drop compared to the flow rate, flow pattern and volumetric concentration of solids. In the present work, air as a gas phase will be added and every resulting flow regime will be analyzed in terms of transport efficiency. Also the previous correlations will be applied and compared to the new experimental results, so as to obtain information of the interaction between the three phases.

Therefore, the current work aims to generate a reliable database of three-phase G/L/S mixtures, flowing in horizontal $30 \mathrm{~mm}$ inner diameter (ID) pipelines for different flow regimes. The G/L/S mixture is composed of air, water and polyethylene particles. The superficial velocity of the slurry phase is in the range of $0-2 \mathrm{~m} / \mathrm{s}$, with low concentrations of solids, in the range of $0-10 \%$ in volume with respect to water, and the air superficial velocity from 0 to $5 \mathrm{~m} / \mathrm{s}$.

\section{Previous Studies}

\subsection{Two-Phase (G/L) Correlations}

The main insight of the Lockhart and Martinelli (1949) correlation is the determination of the frictional pressure gradient (FPG) of the mixture as if each phase was flowing separately in the pipeline. These values are corrected using the two phase multipliers, $\Phi_{f}$ and $\Phi_{g}$ (see Eq. 1). The assumption is that for each phase, there must be a set of hydraulic diameter, phase velocity and friction factor that results in the two-phase FPG. The analysis leads to the postulation that $\Phi_{f}$ and $\Phi_{g}$ are functions of the gas and liquid Reynolds numbers $\left(R e_{f}, R e_{g}\right.$, based on the superficial velocities) and the Lockhart-Martinelli (L-M) parameter $X$, as defined in Eqs. 2 and 3. Empirical curves, for design purposes, were obtained from a wide range of experimental data that confirmed the initial hypothesis.

$$
\begin{gathered}
\left(\frac{\delta P}{\delta z}\right)_{g f}=\Phi_{f}^{2} \cdot\left(\frac{\delta P}{\delta z}\right)_{f}=\Phi_{g}^{2} \cdot\left(\frac{\delta P}{\delta z}\right)_{g} \\
\Phi_{f}^{2}=f\left(R e_{f}, R e_{g}, X\right) \\
X^{2}=\frac{(\delta P / \delta z)_{f}}{(\delta P / \delta z)_{g}}
\end{gathered}
$$

Later, Chisholm (1967) deeply examined the Lockhart and Martinelli's studies, and proposed a simple equation, for design purposes, relating the multiphase multipliers with the Lockhart-Martinelli parameter (LM), $X$ (see Eq. 4). 


$$
\Phi_{f}^{2}=1+C / X+1 / X^{2}
$$

Here, $C$ is a flow regime indicator. It can adopt four different values depending on the flow regime of the phases, because it quantifies in what extent the liquid or gas flows are dominated by turbulence $(t)$ or viscous effects $(v)$.

$$
C=\left\{\begin{array}{llll}
5, & \text { if } R e_{f}<2000 & \text { and } & R e_{g}<2000(v-v) \\
10, & \text { if } R e_{f} \geq 2000 & \text { and } & R e_{g}<2000(t-v) \\
12, & \text { if } R e_{f}<2000 & \text { and } & R e_{g} \geq 2000(v-t) \\
20, & \text { if } R e_{f} \geq 2000 & \text { and } & R e_{g} \geq 2000(t-t)
\end{array}\right.
$$

Since the studies of Chisholm (1967) several authors have tested and validated the LM correlation with the Chisholm simplification, including comparison with other correlations ( $L u$ et al. 2018; Kong et al. 2018; Rahman et al. 2013; Abduvayt et al. 2003; Sun and Mishima 2009; Sassi et al. 2020). In the literature the LM correlation performs reasonably well in predicting the FPG of two phase flows.

\subsection{Two-Phase (G/L) Flow Regime Map}

Several flow regime maps have been proposed by different authors (Baker 1953; Hoogendoorn 1959; Govier and Omer 1962). However there are mainly two flow maps that are generally used. The first one developed by Mandhane et al. (1974) with experimental data obtained from a data base with a wide range of flow regimes and physical properties. The second one developed by Taitel and Dukler (1976) from a theoretical model analysis. The main differences between the two maps are (1) the transition from bubbly to intermittent flow proposed by Mandhane et al. (1974) occurs at superficial velocities of the liquid phase of $4.20 \mathrm{~m} / \mathrm{s}$ independently of the gas superficial velocity, while Taitel and Dukler (1976) state that for higher flow rates of gas the transition occurs at higher rates of liquid. Furthermore, (2) the transition from stratified to intermittent flows in Taitel and Dukler's map requires higher liquid superficial velocities than Mandhane et al.'s map. Finally, (3) Taitel and Dukler (1976) refers to intermittent flows without a distinction between Plug and Slug flows. On the other hand, Mandhane et al. (1974) sets a transition boundary between elongated bubble (plug) and slug flows.

Recently, Kong and Kim (2017) studied the transition boundaries by classifying 263 flow conditions from a detailed flow visualization study using a high-speed video camera. The study includes bubbly, plug, slug, stratified, stratified-wavy and annular flows with their respective transitions. They concluded that the transition from bubbly to intermittent depends on $j_{g}$, as proposed by Taitel and Dukler (1976). In this case they also observed that more gas is required for the transition from plug to slug at $j_{f}$ less than $1 \mathrm{~m} / \mathrm{s}$, and less gas is required for $j_{f}$ above $1 \mathrm{~m} / \mathrm{s}$. In Fig. 1 the regimes maps of Mandhane et al. (1974), Taitel and Dukler (1976) and Kong and Kim (2017) are plotted together for comparison.

\subsection{Two-Phase (L/S) Correlations}

The focus of this research is on heterogeneous slurries. These are composed of coarser particles that segregate in the quiescent state and do not flocculate. These types of slurries are generally turbulent flows, because turbulence is the main suspension mechanism of the particles and at low velocities a stationary bed is formed. Also, the concentration distribution is 
Fig. 1 Regime maps for two-phase flows in horizontal pipelines

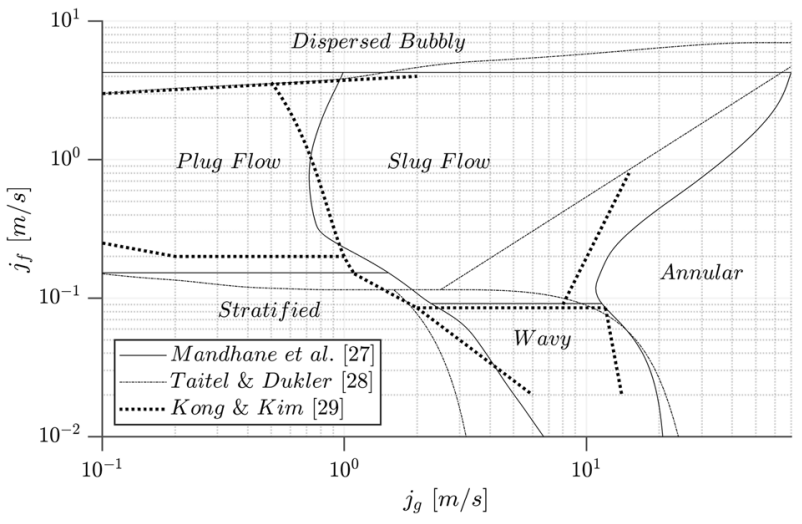

not uniform. This non-uniformity over the cross-section of the pipe often leads to differences between liquid and solid velocities. For pipeline flow of solid-liquid mixtures, there are forces acting on each phase due to the effects of pressure and viscosity, in the presence of the pipe wall. In addition, there are interfacial forces acting on the solids and on the liquid because of the slip velocity. However the net interfacial interaction force per unit volume of mixture must of course be zero.

In Gillies et al. (2004) heterogeneous slurries flowing at high velocities were studied using the Saskatchewan Research Council's (SRC) two-layer model (Gillies and Shook 2000), and incorporating improvements from (Wilson and Sellgren 2003). They divide the system into three superimposed layers. Each layer contributes individually to the frictional pressure loss. The three layers are: the finer particles (type 1 particles), which together with the liquid form a carrier fluid of higher density and viscosity; coarse particles (type 2) that are suspended by the flow turbulence; and particles that are not suspended and constitute a sliding bed (type 3). In this paper only heterogeneous flows are analysed, which are composed of particles from the second category. Accordingly, the FPG is calculated using Eq. 6 and the wall shear stress $\left(\tau_{w}\right)$, which is kinematic, is calculated with Eq. 7

$$
\begin{gathered}
-\left(\frac{d P}{d z}\right)_{f_{s}}=\frac{\tau_{w} S}{A}=\frac{4 \tau_{w}}{D} \\
\tau_{w}=\frac{j_{f s}^{2}}{8}\left(\rho_{f} f_{f}+\rho_{s} f_{s}\right)
\end{gathered}
$$

In Eq. $6 D, S$ and $A$ are the diameter, wetted perimeter and cross sectional area of the pipeline respectively. In Eq. $7 j_{f s}$ is the slurry superficial velocity, $\rho_{f}$ and $\rho_{s}$ are the liquid and particle densities, $f_{f}$ is the Darcy-Weisbach friction factor for the liquid flowing at the slurry velocity. Finally $f_{s}$ is the particle friction factor, it increases the FPG accounting the friction caused by the particle-particle and particle-wall collisions, and it can be computed using Eq. 8, as a function of the linear concentration $\lambda$ (Eq. 9), and the dimensionless particle diameter $d^{+}$, Eq. 10. The linear concentration can be considered a measure of the ratio of the particle diameter to the shortest distance between neighbouring particles. In Eq. 9, $C_{\max }$ is the maximum package concentration (or random close pack) of the solid phase, this is the maximum volume fraction of solid objects obtained when they are packed randomly, and $C_{s}$ is the in-situ volumetric solid concentration. 


$$
\begin{gathered}
f_{s}=0.00132 \cdot \lambda^{1.25}\left[0.15+e^{-0.1 d^{+}}\right] \\
\lambda=\left[\left(\frac{C_{\max }}{C_{s}}\right)^{1 / 3}-1\right]^{-1} \\
d^{+}=\frac{d \cdot j_{f s} \sqrt{f_{f} / 8}}{v_{f}}
\end{gathered}
$$

Several authors studied the FPG of slurries and the SRC two-layer model has shown good accuracy. However, in most of these studies, the analyzed slurries are composed of particles much denser than the fluid. Stutz et al. (2000) investigated the friction factor for slurries with particle density close to that of the water, for analysing solid-liquid coolant systems. In their study they used empirical correlations, based on dimensional analysis. Later, Edelin et al. (2015) studied the energy optimum for the transport of floating particles in pipelines, using models of effective liquid where the liquid-solid mixture is modeled with layers in which different effective properties of the fluid correspond to each layer. To determine the density and viscosity of these layers they considered the shape, size and arrangement of the particles, as well as the fluid in which they are flowing.

\subsection{Three-Phase (G/L/S) Correlations}

Three-phase flows have been reviewed in Rahman et al. (2013). These authors present a summary of the most important studies and their insights including the research from (Scott and Rao 1971), this study has been the first to propose that the Lockhart-Martinelli correlation can be modified to analyze three-phase flows in horizontal pipelines. This insight is advantageous because with the modified L-M correlation, predictions of the mixture FPG can be obtained even if the flow regime is not known, this was not the case in previous three-phase correlations.

The modified Lockhart-Martinelli correlation proposed in Scott and Rao (1971), and later developed in Hatate et al. (1986) and Gillies et al. (1997), estimates the FPG of the three-phase flows by assuming that the slurry FPG can be used instead of the liquid pressure drop in the original correlation. In the work of Scott and Rao (1971) a good correlation performance was obtained using the Durand correlation for calculating the slurry friction gradient. However, the latter correlation is known to provide an accurate prediction only when solid concentration is under $15 \%$. In the study of Hatate et al. (1986) experimental data were correlated within a $30 \%$ error but only for small particles $(d<100 \mu \mathrm{m})$. This denotes the need of an accurate and general estimation of the slurry FPG in order to apply the modified Lockhart-Martinelli correlation.

In summary, in this study, the modified Lockhart-correlation is used to predict the FPG of three-phase flows where the properties of the slurry are first modified according to the two-layer model (Gillies and Shook 2000), assuming that only coarse particles suspended by turbulence are present in the liquid phase. In this way, the LM parameter $(X)$ in Eq. 3 is calculated with the FPG of the slurry. 


$$
X^{2}=\frac{(\delta P / \delta z)_{f s}}{(\delta P / \delta z)_{g}}
$$

Here $(\delta P / \delta z)_{f s}$ is calculated following Eqs. (6-10). Then, the multi-phase multiplier $\left(\Phi_{f_{s}}\right)$ is calculated with Eq. 4, and finally, the FPG for three phase flows is obtained by modifying Eq. 1:

$$
\left(\frac{\delta P}{\delta z}\right)_{g f s}=\Phi_{f s}^{2} \cdot\left(\frac{\delta P}{\delta z}\right)_{f s}
$$

\section{Experimental Set-Up}

A diagram of the experimental facility is represented in Fig. 2, the flow loop has two horizontal and two vertical pipe sections. The set-up is designed to analyze three phase flows, gas-liquid-solid mixtures (G/L/S), and specifically to reproduce the plug and slug flow regimes in horizontal pipelines; and bubbly, slug, churn and annular flow regimes in vertical pipelines. However, the reported and analyzed data in this paper, correspond to experiments for two and three phase, $(\mathrm{G} / \mathrm{L})$ and $(\mathrm{G} / \mathrm{L} / \mathrm{S})$ mixtures, only in the horizontal test section pipeline. A detailed schematic representation of the horizontal test section is shown in Fig. 3.

The gas phase is compressed air, taken from the lab manifold at a pressure of about eight bar, previously filtered and dried. The air pressure is set at four bar at the entrance of the rotameters with a reduction valve. The air flow rate is measured using two Omega valve-rotameters with an accuracy of $2 \%$ and $3 \%$ respectively. Air is introduced in the test section through a "Y" junction.

Generic tap water is used as the liquid phase. It is stored in a $100 \mathrm{~L}$ slurry-tank. The solid phase consists of polypropylene pellets, with density $\rho_{s}=866 \mathrm{~kg} / \mathrm{m}^{3}$, the measured

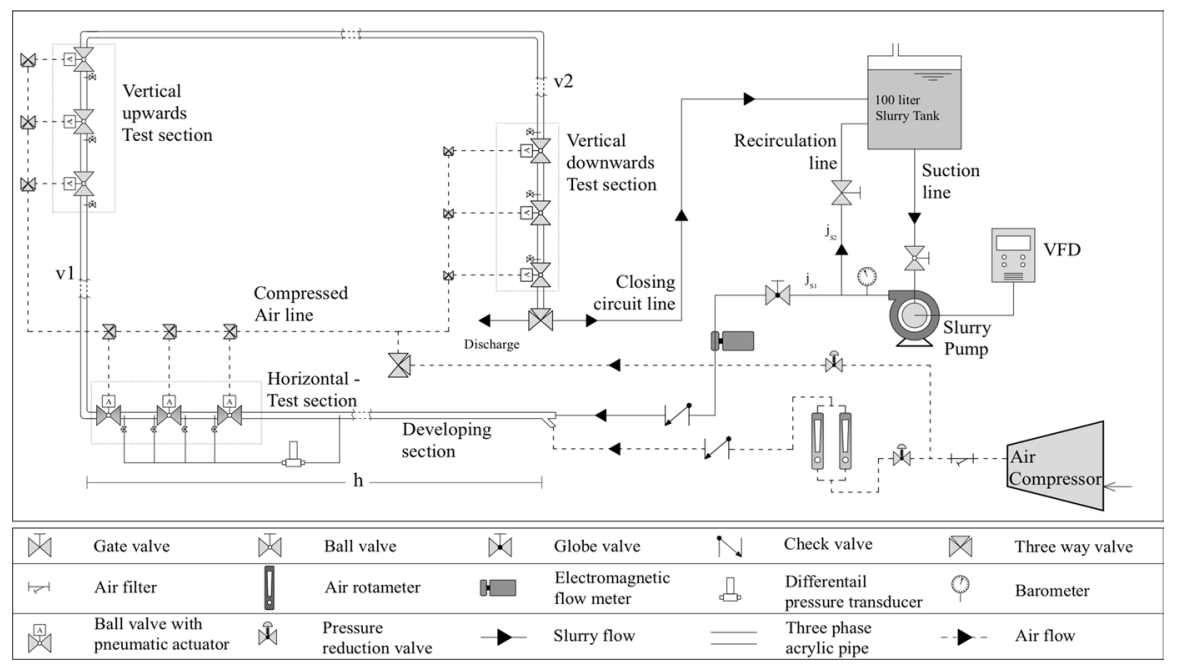

Fig. 2 Schematic of the experimental facility (not to scale) 


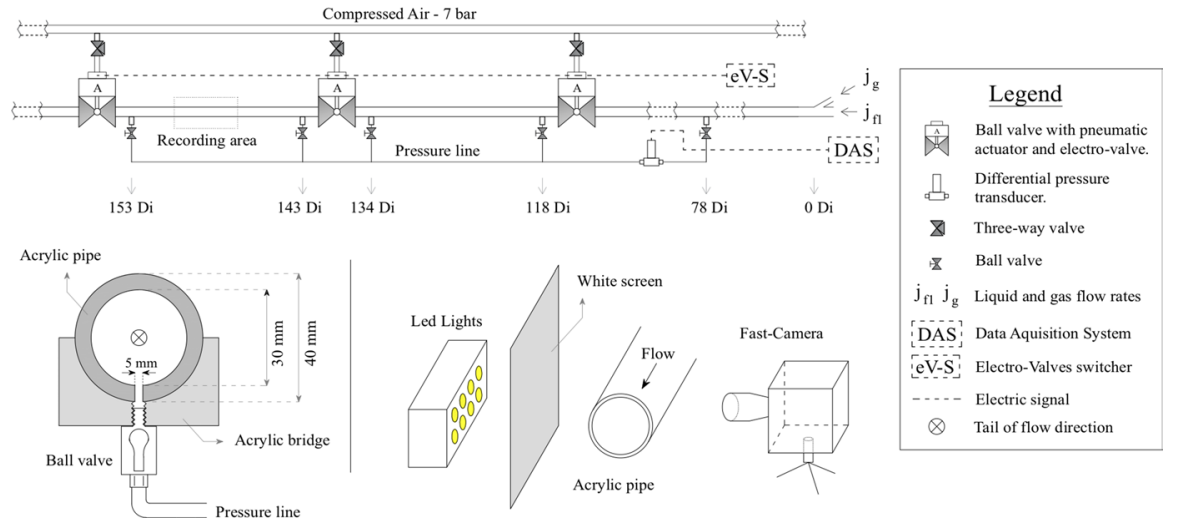

Fig. 3 Schematic of the horizontal test section (not to scale)

maximum packing concentration is $C_{\max }=0.585$. It was measured by submerging the particles in ethanol $\left(\rho_{\text {ethanol }}=789 \mathrm{~kg} / \mathrm{m}^{3}\right)$. The particle diameters are between 1 and $2 \mathrm{~mm}$ according to the sieves used for the diameter separation. The pellets are added in the slurry tank with a specific concentration and the mixture (slurry) is pumped with a $5.5 \mathrm{~kW}$ Weir model AB80 centrifugal slurry-pump. The main flow, $j_{f 1}$, is measured with an Isoil MS2500 electromagnetic flow meter with an accuracy of $\pm 0.8 \%$, and then delivered to the test section (note that $j_{f 1}=j_{f}$ in gas-liquid two-phase flow, and $j_{f 1}=j_{f s}$ in three-phase flow). A secondary flow, $j_{f 2}$, is recirculated to the slurry tank with two purposes, (i) to ensure the mixing of the slurry by generating turbulence inside the tank, and (ii) to be able to measure a wider spectrum of slurry flow rates together with a Weg variable frequency drive (VFD), which controls the slurry-pump.

The slurry mixture is homogenized due to the turbulence in the tank. However, as particles float in water, solid percentage measurements have been made within the test section. For this, the slurry mixture is circulated through the facility and the fast-closing valves (see Fig. 3) are closed simultaneously. The volume fractions of liquid and solid phases, trapped between the valves, are extracted and measured in test tubes. For a mixture of $5 \%$ of solid concentrations (in volume with respect to water) 10 measurements were performed for 4 different flow rates, adding a total of 40 measurements, the solid percentage is $5.0 \pm 0.5 \%$. For mixtures of $10 \%$ the same process was followed. In this case the mean value was $10 \pm 1 \%$.

In this study, only horizontal flows are analyzed, the three-phase horizontal test section consist of $30 \mathrm{~mm}$ ID straight transparent acrylic pipes with a total length of 60 diameters and a previous segment of 110 diameters in order to ensure the complete development of the flow (see Fig. 3). An analysis of the development of the flow using the pressure transducers has been done in a previous study (Sassi et al. 2020).

The flow visualization is performed using a Photron Mini UX100 fast camera. To identify the flow regime each test condition is visualized, recording $5 \mathrm{~s}$ at 3200 frames per second. Images have $1280 \times 560$ pixels with a spatial resolution of $70 \mu \mathrm{m} / \mathrm{pixel}$. Images are taken with back led illumination and using a translucent white screen to diffuse light. The detail of the visualization set-up is shown in Fig. 3. Each operating condition is carefully visually analyzed to determine if it corresponds to plug, slug or transition flow regime. 
Two Omega differential pressure transducers are connected to the horizontal test section in order to measure the frictional pressure loss inside the pipeline. The pressure lines are connected to 5 points of the acrylic pipes, as detailed in the bottom left corner of Fig. 3 by coupling an acrylic "bridge" with a threaded hole where a small ball valve is placed. Different segments can be measured by selecting the corresponding combination of valves. Caution is needed to avoid air in the pressure lines, as it would distort the measurements. An Agilent 34970A data acquisition system is used to carry out the measurements at $20 \mathrm{~Hz}$. Further details of the experimental facility can be found in Sassi et al. (2020).

Temporal pressure fluctuations on intermittent flow regimes are relatively large, as reported by Spedding and Spence (1993). In the present study the pressure drop per unit length is calculated by averaging the temporal signal of pressure drop between different test sections. For each test condition the differential pressure is measured at $20 \mathrm{~Hz}$ during $120 \mathrm{~s}$ between four different distances, together with the gauge pressure. As an example, Fig. 4 shows the signals from the pressure transducers of two flow regimes, corresponding to three-phase flows with $10 \%$ concentration of solids by volume, at two axial positions, $L=118 I D$ (blue) and $L=153 I D$ (red). The measurements in Fig. 4a correspond to plug flow, with standard deviations of $1.7 \%$ (blue) and $1.4 \%$ (red), while the gauge pressure from Fig. 4b, corresponds to slug flow, with standard deviations of $2.2 \%$ (blue) and $1.7 \%$ (red). Despite the fluctuations, specially in slug flows, it can be seen that, for both flow regimes, the signals range around the mean value with relative small values of standard deviation.

In order to ensure the accurate estimation of the average pressure at the corresponding sections, single-phase water flow measurements were performed and compared with the theoretical curve of the Darcy-Weisbach equation (Eq. 13). The Darcy-Weisbach friction factor, $f$, is calculated with the Colebrook-White relation (Eq. 14). Figure 5 shows the comparison. The mean deviation of the twelve performed measurements is $4 \%$.

$$
\left(\frac{\delta P}{\delta z}\right)_{f}=f \cdot \frac{\rho}{2} \cdot \frac{j_{f}^{2}}{D}
$$

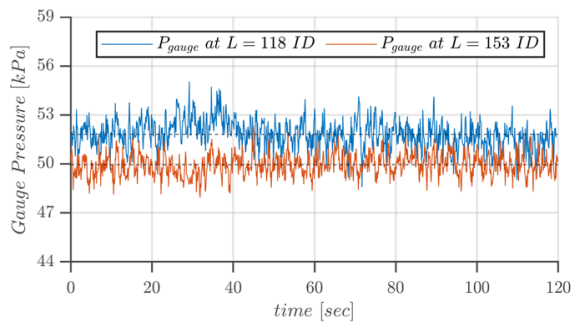

(a) Plug flow, $j_{f s}=1.70 \mathrm{~m} / \mathrm{s}$ and $j_{g}=0.43 \mathrm{~m} / \mathrm{s}$.

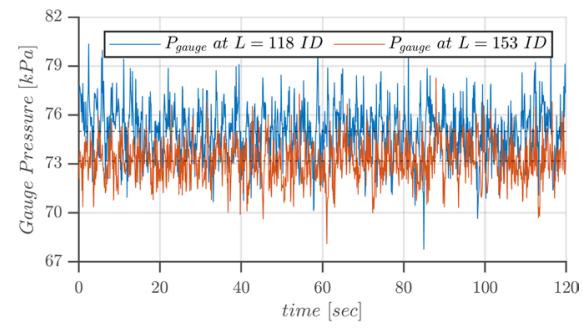

(b) Slug flow, $j_{f s}=1.70 \mathrm{~m} / \mathrm{s}$ and $j_{g}=2.17 \mathrm{~m} / \mathrm{s}$.

Fig. 4 Temporal fluctuations on the gauge pressure measurements for three-phase flow, 10\% loading of solids 
Fig. 5 Comparison of experimental frictional pressure gradient for water flow and Darcy-Weisbach theoretical curve with $\epsilon=1.0 \times 10^{-5} \mathrm{~m}$ (thermoplastics)

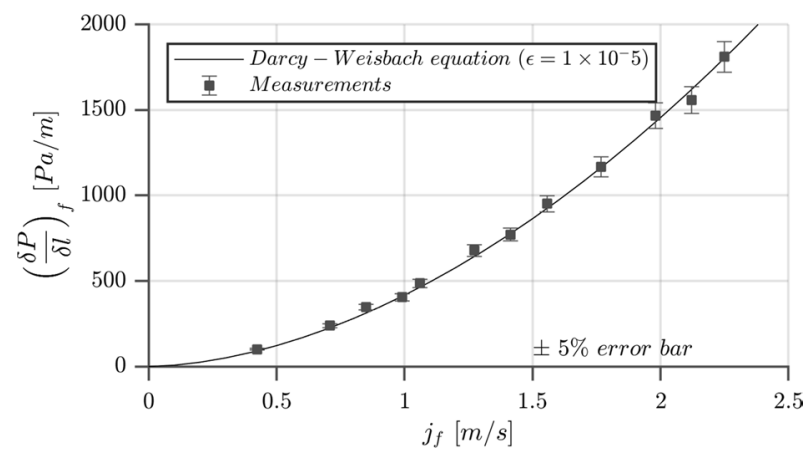

$$
\frac{1}{\sqrt{f}}=-2 \log _{10}\left(\frac{k / D}{3,7}+\frac{2,51}{\operatorname{Re} \sqrt{f}}\right)
$$

A total of 134 test conditions were measured in the experimental facility. 62 were specifically chosen to analyze the transition between plug and slug flow regimes. Here, the gas and slurry velocities were set according to the flow visualization to determine precisely the flow transition. For the analysis of the FPG, 24 different operating conditions were selected for the three mixtures (two-phase and, 5\% and $10 \%$ of solid loading), adding a total of 72 measurements. A summary of the performed measurements is shown in Table 1.

Table 1 Summary of experimental data

\begin{tabular}{ccccc}
\hline Objective & Solid \% & $j_{g}[\mathrm{~m} / \mathrm{s}]$ & $j_{f s}[\mathrm{~m} / \mathrm{s}]$ & \# points \\
\hline Flow & $2 \mathrm{P}$ & $0.4-1.2$ & $0.6-2$ & 25 \\
Transition & $5 \%$ & $0.7-1.5$ & $0.6-2$ & 19 \\
(Visualization) & $10 \%$ & $0.7-1.5$ & $0.6-2$ & 18 \\
\hline Sub-total & \multicolumn{5}{c}{0.4} & 62 \\
\hline & & 0.4 & 1 & \\
Frictional & $2 \mathrm{P}$ & 0.6 & 1.4 & $(3 \times 6 \times 3)$ \\
Pressure & $5 \%$ & 1.4 & 1.7 & 54 \\
Gradient & $10 \%$ & 2 & & \\
\cline { 3 - 5 } & & 2.5 & 1 & $(3 \times 3 \times 2)$ \\
& & 3.5 & 1.4 & 18 \\
\hline Sub-total & & 4.5 & & 72 \\
\hline Total & & & &
\end{tabular}




\section{Results and Discussion}

The results of this experimental study are discussed in two main sections: Sect. 4.1 Flow regime identification, where flow images and the flow regime map are analyzed; and Sect. 4.2 Frictional pressure gradient analysis, where the experimental data is compared with available correlations of the literature.

\subsection{Flow Regime Identification}

The fast camera is used to visualize and identify the flow regime. Videos are recorded at an axial location of $L / D=150$ from the inlet to analyze fully developed flow conditions. Videos are taken for several runs within plug, plug-to-slug transition and slug flows in order to identify the transition boundaries.

Figure 6 shows three different flow regimes of two-phase flow. In Fig. 6a a plug flow elongated bubble is shown. It can be seen that this flow regime is characterized by elongated gas plugs that move along the top of the pipe, which generally presents a round nose. Gas plugs displace the liquid phase towards the wall of the pipe producing a very thin film of liquid in between. From the figure it can be inferred an important shear force between the two phases, as evidenced by the slight stripes in the thin film, and the waves produced at the bottom of the main body of the plug bubble. Finally, plug bubbles show elongated tails in the very upper portion of the pipe followed by liquid regions with few bubbles that

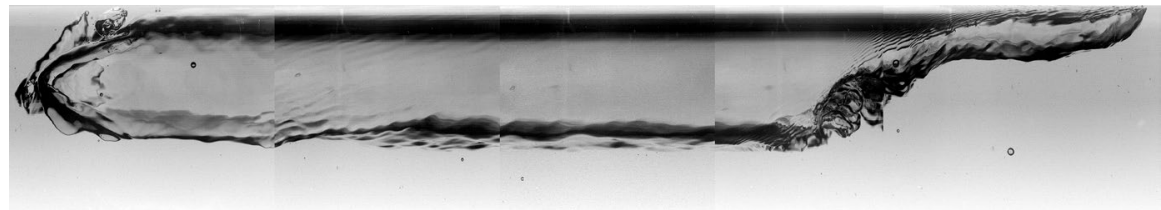

(a) Plug flow elongated bubble; $j_{g}=0.40 \mathrm{~m} / \mathrm{s}, j_{f s}=1.0 \mathrm{~m} / \mathrm{s}$.

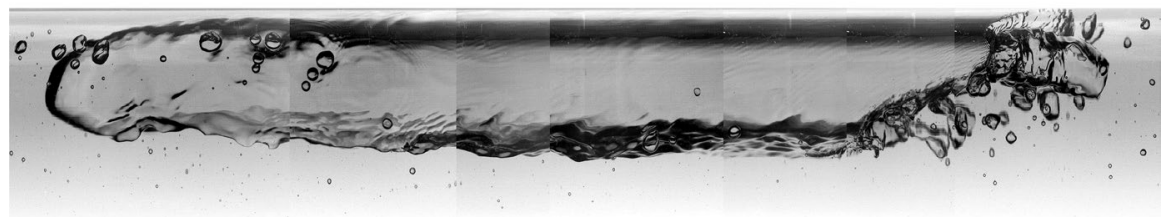

(b) Plug to Slug Transition elongated bubble; $j_{g}=1.0 \mathrm{~m} / \mathrm{s}, j_{f s}=1.0 \mathrm{~m} / \mathrm{s}$

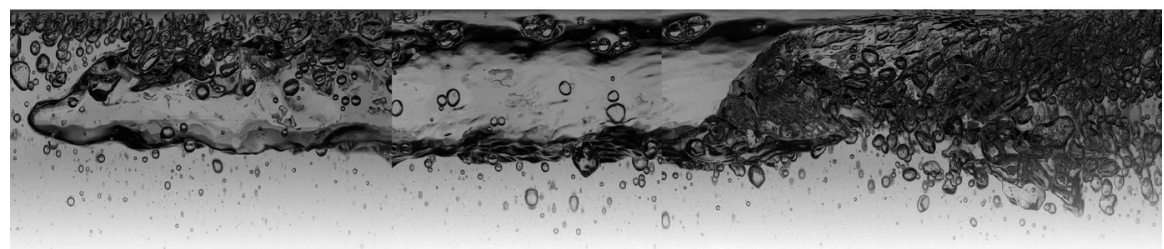

(c) Slug flow elongated bubble; $j_{g}=1.0 \mathrm{~m} / \mathrm{s}, j_{f_{s}}=2.0 \mathrm{~m} / \mathrm{s}$

Fig. 6 Plug, transition and slug flow reconstructed bubbles for two-phase flow (flowing from right to left) 
detached from the tail. Some of these observations, among some other characteristics on plug flows, can also be found in Talley et al. (2015a).

In Fig. 6b a plug-to-slug transition bubble is shown. It can be seen how the shear force between the two phases is increased as more stripes are found in the thin film and the bottom interface, which appears more wavy, even producing the detachment of small droplets inside the gas bubble. Also as a result of the increase in the shear force, the tail breaks into smaller bubbles that travel behind.

Finally, in slug flows (see Fig. 6c) the elongated bubbles move forward faster than the liquid phase, and they are followed by bubble clusters. The increase of the relative velocity yields higher shear forces, and consequently, these bubbles show a sharper nose compared with plug bubbles and a chaotic tail with continuous detachment of small bubbles. Following the tail, bubble clusters are spread within the volume of the pipe due to the higher turbulence of the flow that suspends the bubbles in the flow. The presence of the bubble clusters is the main characteristic that distinguish slug from plug flows, as highlighted in Talley et al. (2015a). Because these bubbles are dispersed in a turbulent flow, there is a relative motion between the air bubble and the water. This relative velocity between the phases induces even more turbulence in the liquid phase, specifically in the wake of the bubble. This phenomenon is known as the bubble-induced turbulence. More details on the phenomena can be found in Rzehak and Krepper (2013). Further, the existence of a vortex following slug bubbles is observed while in plug flows these are not found. The result is a very large interface between the phases with high turbulence in slug flows. Therefore, this regime is more appropriate for heat and mass transfer applications.

The same identification criterion is used for three-phase flows. In Fig. 7, three images for three-phase flows with 5\% of solid concentration are shown. Figure 7 a corresponds to plug flow, Fig. $7 \mathrm{~b}$ to plug-to-slug transition, and Fig. 7c to slug flow.

Again, the main difference stands in the liquid plugs or slugs between the elongated bubbles. In plug flows there are barely air bubbles between the plug bubbles and mostly

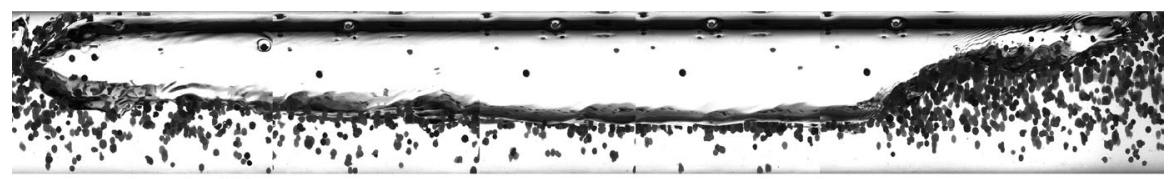

(a) Plug flow elongated bubble; $j_{g}=0.40 \mathrm{~m} / \mathrm{s}, j_{f s}=1.0 \mathrm{~m} / \mathrm{s}$.

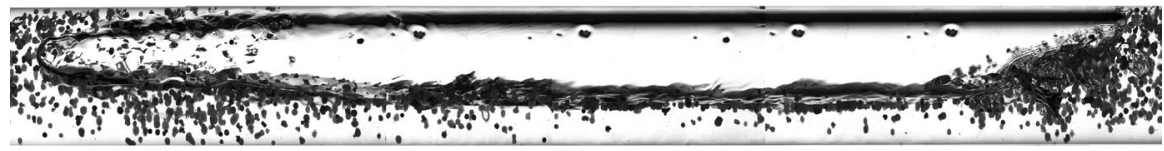

(b) Plug-to-Slug Transition elongated bubble; $j_{g}=1.0 \mathrm{~m} / \mathrm{s}, j_{f s}=1.0 \mathrm{~m} / \mathrm{s}$

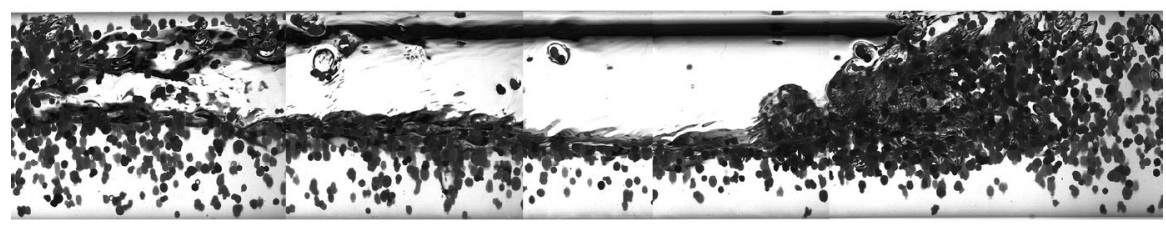

(c) Slug flow elongated bubble; $j_{g}=1.0 \mathrm{~m} / \mathrm{s}, j_{f s}=2.0 \mathrm{~m} / \mathrm{s}$

Fig. 7 Plug, transition and slug flow reconstructed bubbles for three-phase flows with 5\% of solid concentration (flowing from right to left) 
there are only solid particles. On the contrary, in slug flows there are many air bubbles among the solid particles. Plug flows also show a higher concentration of particles at the top of the pipeline in comparison to slug flows. This is mainly because of the higher turbulence on the latter flows, with higher transverse velocities, that effectively suspend the solid particles in the liquid phase.

Videos have been recorded for 62 different test conditions including plug, plug-to-slug transition and slug regimes for air-water two-phase flows and air-water-polypropylene three-phase flows with 5 and $10 \%$ of solid concentrations related to the liquid phase. Each condition was thoroughly visually analyzed in order to determine the corresponding regime and unveil the influence of solid concentration on the transition boundaries.

In Fig. 8 the section of the regime map covered in the present study is displayed. Transition boundaries between plug and slug proposed by Mandhane et al. (1974) and Kong and Kim (2017) for two-phase flows are indicated in the legend, as well as the proposed ones in the current study, for three-phase flows. Each test condition is plotted with a marker in the map. White marks correspond to air-water two-phase runs, grey marks to three-phase runs with 5\% loading of solids and black marks to $10 \%$ loading. The flow regime visualized for each run is indicated with the shape of the markers, triangles for plug flow, diamonds for plug-to-slug transition and squares for slug flow.

From the visual analysis of the two-phase runs it is observed that transition zones are wide, and they can hardly be expressed with a line. In any case, our measurements agree with the observations of Kong and Kim (2017) regarding the plug-to-slug transition, where they claim that less air is required to reach slug flow at $j_{f}$ above $1 \mathrm{~m} / \mathrm{s}$ and vice versa.

For three-phase flows, it is observed that the accumulation of particles in the tail of plug flows, delays the small bubble detachment for increasing relative velocities, thus the transition from plug to slug flow is displaced towards higher gas superficial velocities.

In plug flows, there are large slurry regions between the plug bubbles. However, collision and eventually coalescence of the gas bubbles is observed frequently. Regular plug bubbles are wider in the nose region. This widening induces an acceleration in the slurry that, at the same time, produces a wave. While this wave is propagating towards the tail of the bubble, it may grow larger than the depth of the plug tail. If so, the tail is cut off and a smaller bubble stands between the two large plug bubbles. As the solid particles remain in the liquid phase (they do not cross the liquid-gas interface) after the detachment is completed an accumulation of particles is observed around the detached bubbles, and these smaller bubbles generally coalesce with the next plug bubble. This mechanism is known as drag induced coalescence, where larger bubbles overtakes the smaller ones due to relative motion (Talley et al. 2015a). In Fig. 9 a sequence of the coalescence process of a plug bubble with a smaller bubble is shown for a three-phase, 5\% loading plug regime flow,

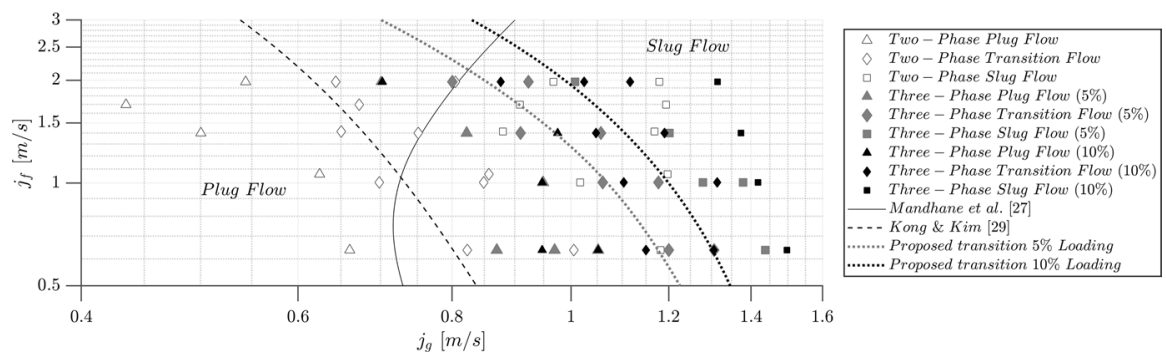

Fig. 8 Flow regime map 


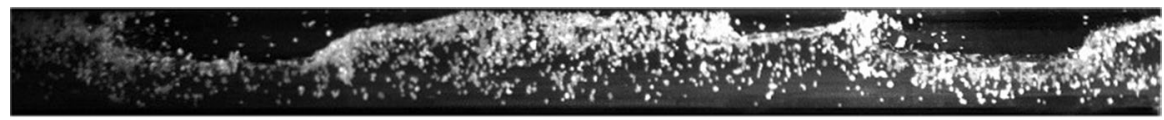

(a) $t=0 \mathrm{~ms}$ - plug bubble (right) reaching detached small bubble.

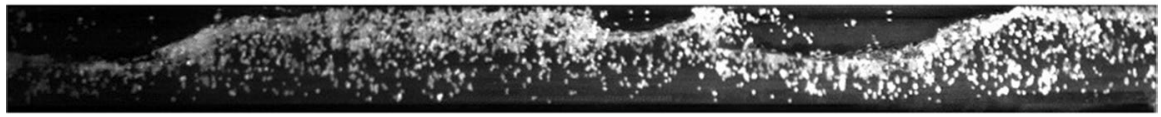

(b) $t=37.5 \mathrm{~ms}$ - accumulation of solid phase (white dots) between the bubbles.

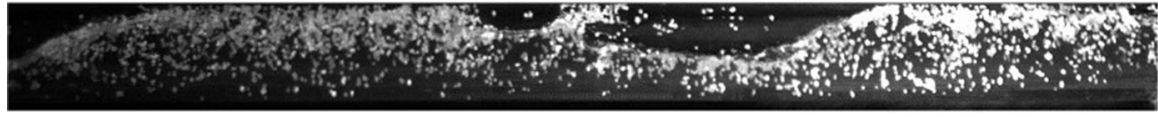

(c) $t=71.3 \mathrm{~ms}$ - displacement of solid phase around the nose of plug bubble.

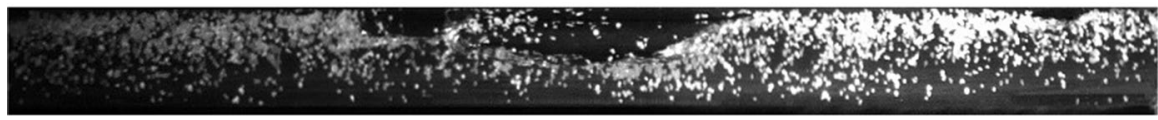

(d) $t=101.5 \mathrm{~ms}$ - the interface between bubbles gets thinner.

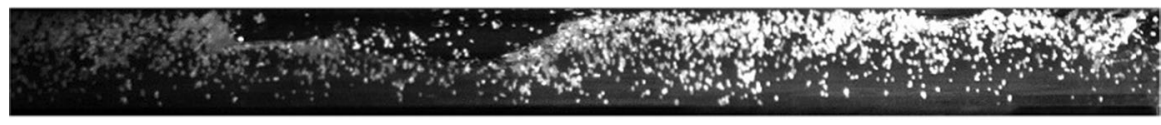

(e) $t=138.1 \mathrm{~ms}$ - coalescence, the interface collapses.

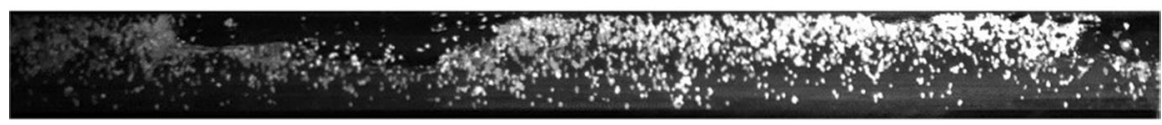

(f) $t=153.8 \mathrm{~ms}$ - reshape of the bubble after coalescence.

Fig. 9 Coalescence sequence of a plug bubble and a smaller one, $j_{f s}=1.06 \mathrm{~m} / \mathrm{s}, j_{g}=0.4 \mathrm{~m} / \mathrm{s}$ and $C_{s}=0.05$, flow from right to left

with $j_{f s}=1.06 \mathrm{~m} / \mathrm{s}$ and $j_{g}=0.4 \mathrm{~m} / \mathrm{s}$. In Fig. 9 a the plug bubble on the right is reaching a smaller one, previously detached from the large plug bubble on the very left of the image. In Fig. 9b, $\mathrm{c}$ an interface between the two bubbles is developed. An accumulation of solid particles between the two interfaces is observed (white dots). The solid particles are displaced around the nose of the plug bubble and some of them get attached to the pipe wall. Later in Fig. 9d the interface gets thinner, while the solid particles are still being displaced, until the interface breaks in Fig. 9e. Finally, (Fig. 9f) the large bubble formed from the coalescence moves forward reshaping into the plug bubble shape and the solid particles attached to the pipe wall are dragged by the slurry.

In slug flows, the presence of the small bubbles in the slurry regions between the slug (large) bubbles can be explained with the same mechanism as in plug bubbles. This is by the break up of the tail region induced by the waves. But another break up process is observed. This other process is linked with the relative velocity between the gas and slurry phases. As the relative velocity increases higher shear stresses are generated and detachment of several small bubbles is observed. These phenomena are illustrated in Figs. 6 and 7. It can be seen that as the relative velocity increases the number of bubbles 


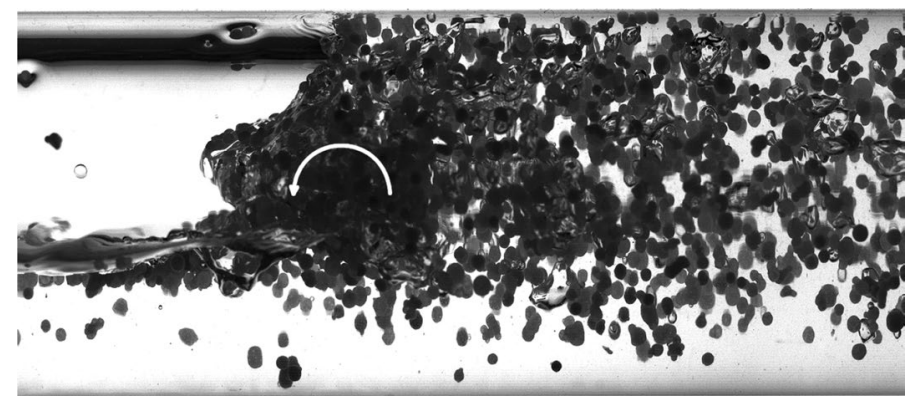

Fig. 10 Animation of a vortex located next-to the tail of a slug elongated bubble, $j_{f s}=1 \mathrm{~m} / \mathrm{s}, j_{g}=1 \mathrm{~m} / \mathrm{s}$ and $C_{s}=0.1$

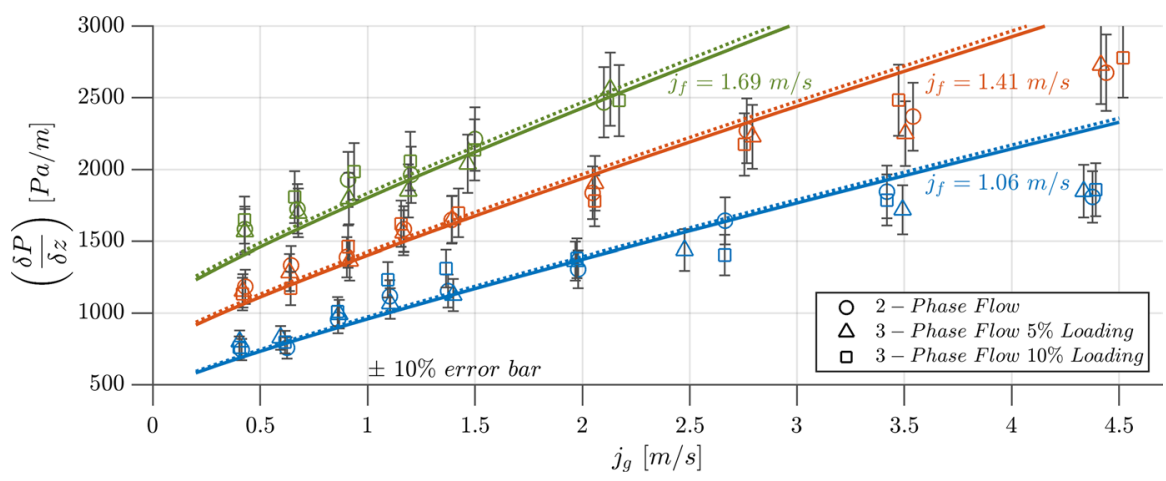

Fig. 11 Performance of the modified Lockhart-Martinelli correlation

also increases until the density of small bubbles is so high that the bubbles become indistinguishable. Finally, it is also appreciable from the videos, that an anticlockwise vortex follows the slug elongated bubbles (Fig. 10). These vortices produce a continuous bubble detachment from the lower tip of the slug bubble, and they also contribute to break the upper part of the tail, observing the effect of a breaking wave in the tail of the bubble. During the break some droplets are ejected into the air bubble and several small bubbles are detached from it. It is also appreciable that the breaking wave goes through the bottom interface producing a vertical downward velocity in the tail of the bubble and it helps the mixing of the phases.

\subsection{Frictional Pressure Gradient Analysis}

To calculate the FPG the modified L-M correlation has been used. In this correlation the slurry (L/S) is treated as the 'liquid' phase, but correcting the physical properties of the slurry as proposed by the Saskatchewan Research Council's Pipe Flow Technology Centre (Gillies and Shook 2000). For this analysis 72 run tests where performed, measurements of pressure drop for two and three-phase flows are plotted together in Fig. 11 against the air superficial velocity $\left(j_{g}\right)$. Circles indicate two-phase runs, triangles and squares three-phase runs with $5 \%\left(C_{s}=0.05\right)$ and $10 \%\left(C_{s}=0.1\right)$ solid loading respectively. The continuous 
lines represent the predictions obtained by applying the L-M correlation for the different liquid superficial velocities, and the dotted lines are obtained by applying the modified L-M correlation for three-phase flows with $10 \%$ loading of solids. Finally, the colors of the curves and markers indicate the liquid (or slurry in 3-phase flows) superficial velocity, $j_{f}=1.06, j_{f}=1.41$ and $j_{f}=1.69 \mathrm{~m} / \mathrm{s}$ are represented by blue, red and green colors respectively.

Although the theoretical curves fit the experimental data relatively well, a general tendency to under-estimate the pressure drop for lower values of superficial gas velocity $\left(j_{g}\right)$ and over-estimate it for higher values of $j_{g}$ is appreciable. Note that the curves corresponding to the same liquid or slurry superficial velocities of two and three-phase $\left(C_{s}=0.1\right)$ flows are in close proximity, as a $10 \%$ load of solid particles, of such density, barely induces an effect on the FPG. This is also noticeable by looking at the experimental data. According to the model (modified LM), the increase in pressure loss due to the incorporation of $10 \%$ in volume of solids is below $2 \%$. A $10 \%$ increase would be reached by adding $44 \%$ in volume of solids with this density $\left(\rho_{s}=866 \mathrm{~kg} / \mathrm{m}^{3}\right)$, however, it would be necessary to verify if the assumption of fully suspended particles of Sect. 2.3 is still valid, or if particles of type 3 (forming a sliding bed, in this case, on the top of the pipe) are present. Another parameter that influences the FPG is the density of the solid phase, when assuming a solid phase with a density of $1500 \mathrm{~kg} / \mathrm{m}^{3}$, the concentration of solid should be $38 \%$ in volume to achieve a 10\% increase in the FPG. Here again, the hypothesis of Sect. 2.3 should be verified, as type 1 and 3 particles would probably be present in the flow.

When applying the Chisholm (1967) simplification, the multi-phase multiplier can be plotted as a function of the L-M parameter, $X$. In two-phase flows, $X$ is calculated with Eq. 3 using the liquid and gas superficial velocities, while in three-phase flows the slurry and gas superficial velocities are used. This means that the difference between two and three-phase flows stands in how the liquid or slurry FPGs are calculated, and so, once $X$ is calculated, both phases can be plotted together in a $\Phi(X)$ plot. This is shown in Fig. 12. The experimental data is plotted together with the predicted values using two values of $C$. The shape of the markers indicate the flow regime of the run where triangles, diamonds and squares represent plug, transition and slug flows respectively. The color of the markers refers to the type of flow. In this way blue, red and green stands for two-phase, three-phase

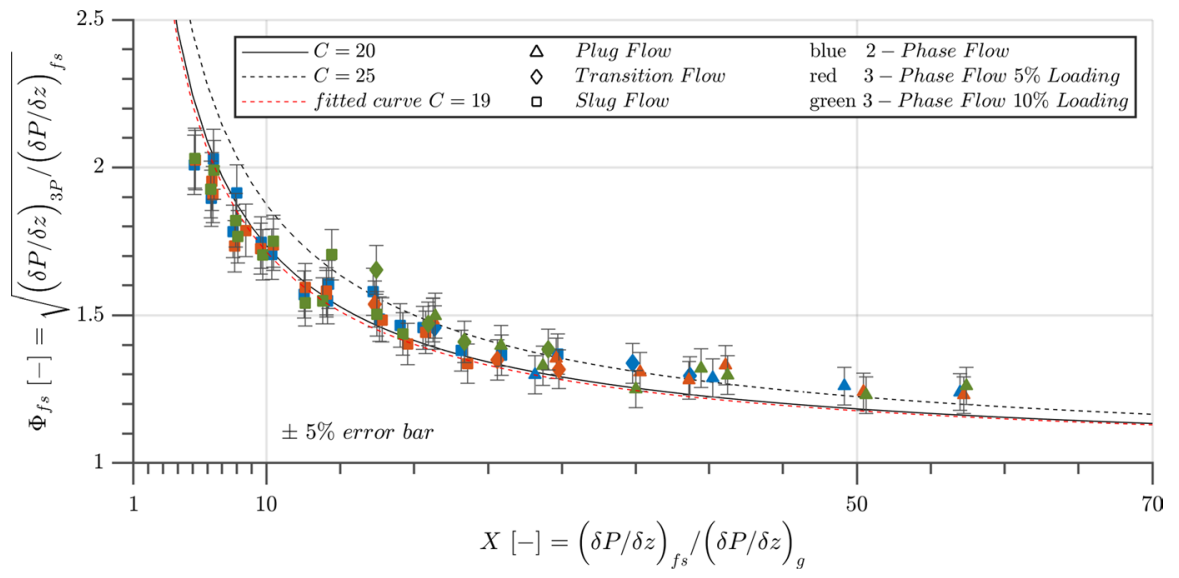

Fig. 12 Multi-phase multiplier against Lockhart-Martinelli parameter 
with $5 \%$ and $10 \%$ of solid concentration respectively. The black plotted curves are the predicted values of $\Phi$ applying the Chisholm simplification. The continuous line is obtained with a value of $C=20$ which is the original proposed value of $C$ for mixtures flowing in a turbulent-turbulent regime (Chisholm 1967). A value of $C=20$, was also found to perform within $\pm 3 \%$ in the experimental data from (Lu et al. 2018). The dashed line in Fig. 12 is obtained with $C=25$, this value was proposed by some authors in the literature (Talley et al. 2015b; Kong and Kim 2017).

The experimental data have also been fitted obtaining the red dashed line that corresponds to a value of $C=19$. The average absolute difference of the experimental data with the curves in Fig. 12 for $C=20, C=25$ and $C=19$ are within 4\%, 6\% and 4\% respectively. This indicates that the LM and modified-LM correlations performs well for both, two-phase and three-phase flows respectively. As a final remark, in Talley et al. (2015b); Kong and Kim (2017) where a value of $C=25$ was found to fit best, bubbly flows were analyzed. These flows correspond to higher values of $X$ (from 60 to 600). It can be seen from the experimental data plotted in Fig. 12 that a value of $C=25$ fits better the points with higher $X$. This is in concordance with Talley et al. (2015b), Kong and Kim (2017), and denotes that $C$ should not be a constant value.

\section{Conclusions and Future Work}

A new experimental facility was used to perform measurements of two- (air-water) and three-phase (air-water-polypropylene) flows. A total of 134 different runs including plug, slug and plug-to-slug flow regimes were carried out.

The flow regimes for 62 different test conditions were deeply analyzed with fast-camera videos in order to identify the transition boundary between plug and slug flows regimes. The transition for two-phase flow agreed well with the flow regime map proposed by Kong and Kim (2017), and a strong dependency of the boundary with the solid concentration was found for three-phase flows. For a specific slurry velocity $\left(j_{f s}\right)$ higher flow rates of the gas phase are needed to reach the boundary as the solid concentration increases.

The pressure drop was analyzed for 72 test conditions. Two-phase measurements were compared with the Lockhart-Martinelli correlation, while the three-phase measurements were compared with the modified Lockhart-Martinelli correlation, incorporating the SRC Pipe Flow model. Both correlations performed adequately as the predicted values of the pressure drop were generally in concordance with the experimental data. It has been noted that for solid concentrations of 5 and 10\% there is a small (almost null) effect on the pressure drop, and according to the theoretical model (modified LM) it is not expected to obtain meaningful increments in pressure drops until reaching more than $30 \%$ of solid concentrations. In any case, experiments with higher concentrations should be performed in order to validate this observation.

Further measurements will be performed to analyze the effect of higher concentration of solids and the influence of the solid density, both to define the effect on the transition from plug to slug regimes and to analyze its effect on the frictional pressure gradient. Also a wider area of the regime map is expected to be covered, analyzing the transition boundary from plug and slug to bubbly flows.

Funding This study has received funding from the European Union's Horizon 2020 research and innovation programme under the Marie Sklodowska-Curie Grant Agreement No. 713679; from the Spanish government under the Grant DPI2016-75791-C2-1-P; and from Generalitat de Catalunya under Grant 
2017-SGR-01234. These supports are gratefully acknowledged. We also appreciate the great help of the lab technician Jordi Iglesias

\section{Compliance with ethical standards}

Conflict of interest The authors declare that they have no conflict of interest.

Open Access This article is licensed under a Creative Commons Attribution 4.0 International License, which permits use, sharing, adaptation, distribution and reproduction in any medium or format, as long as you give appropriate credit to the original author(s) and the source, provide a link to the Creative Commons licence, and indicate if changes were made. The images or other third party material in this article are included in the article's Creative Commons licence, unless indicated otherwise in a credit line to the material. If material is not included in the article's Creative Commons licence and your intended use is not permitted by statutory regulation or exceeds the permitted use, you will need to obtain permission directly from the copyright holder. To view a copy of this licence, visit http://creativecommons.org/licenses/by/4.0/.

\section{References}

Abduvayt, P., Arihara, N., Manabe, R., Ikeda, K.: Experimental and modeling studies for gas-liquid twophase flow at high pressure conditions. J. Jpn. Pet. Inst. 46(2), 111-125 (2003). https://doi.org/10.1627/ jpi.46.111. ISSN 13468804

Baker, O.: Design of pipelines for the simultaneous flow of oil and gas. Soc. Pet. Eng. (1953). https://doi. org/10.2118/323-G

Beggs, H.D., Brill, J.P.: A study of two-phase flow in inclined pipes. J. Pet. Technol. 25(5), 607-617 (1973)

Bello, O.O., Reinicke, K.M., Teodoriu, C.: Particle holdup profiles in horizontal gas-liquid-solid multiphase flow pipeline. Chem. Eng. Technol. 28(12), 1546-1553 (2005). https://doi.org/10.1002/ ceat.200500195. ISSN 09307516

Chisholm, D.: A theoretical basis for the Lockhart-Martinelli correlation for two-phase flow. Int. J. Heat Mass Transf. 10, 1767-1778 (1967)

Cihang, L., Kong, R., Qiao, S., Larimer, J., Kim, S., Bajorek, S., Tien, K., Hoxie, C.: Frictional pressure drop analysis for horizontal and vertical air-water two-phase flows in different pipe sizes. Nucl. Eng. Des. 332(September 2017), 147-161 (2018). https://doi.org/10.1016/j.nucengdes.2018.03.036. ISSN 00295493

Dukler, A.E., Wicks, M., Cleveland, R.G.: Frictional pressure drop in two-phase flow: an approach through similarity analysis. Am. Inst. Chem. Eng. J. 10(1), 44-51 (1964). https://doi.org/10.1002/aic.69010 0118. ISSN 0001-1541

Edelin, D., Czujko, P.C., Castelain, C., Josset, C., Fayolle, F.: Experimental determination of the energy optimum for the transport of floating particles in pipes. Exp. Therm. Fluid Sci. 68, 634-643 (2015). https://doi.org/10.1016/j.expthermflusci.2015.06.018. ISSN 08941777

Gillies, R.G., Shook, C.A.: Modelling high concentration slurry flows. Can. J. Chem. Eng. 78(August), 709-716 (2000). https://doi.org/10.1002/cjce.5450780413. ISSN 00084034

Gillies, R.G., McKibben, M.J., Shook, C.A.: Pipeline flow of gas, liquid and sand mixtures at low velocities. J. Can. Pet. Technol. 36(9), 16-17 (1997)

Gillies, R.G., Shook, C.A., Xu, J.: Modelling heterogeneous slurry flows at high velocities. Can. J. Chem. Eng. 82(October), 1060-1065 (2004). https://doi.org/10.1002/cjce.5450820523. ISSN 00084034

Govier, G.W., Omer, M.M.: The horizontal pipeline flow of air-water mixtures. Can. J. Chem. Eng. 40(3), 93-104 (1962). https://doi.org/10.1002/cjce.5450400303

Hatate, Y., Nomura, H., Fujita, T., Tajiri, S., Ikari, A.: Gas holdup and pressure drop in three-phase horizontal flows of gas-liquid-fine solid particles system. J. Chem. Eng. Jpn. 19(4), 330-335 (1986). https:// doi.org/10.1252/jcej.19.330. ISSN 00219592

Hoogendoorn, C.J.: Gas-liquid flow in horizontal pipes. Chem. Eng. Sci. 9(4), 205-217 (1959). https://doi. org/10.1016/0009-2509(59)85003-X. ISSN 0009-2509

Kago, T., Saruwatari, T., Kashima, M., Morooka, S., Kato, Y.: Heat transfer in horizontal plug and slug flow for gas-liquid and gas-slurry systems. J. Chem. Eng. Jpn. 19(2), 125-131 (1986). https://doi. org/10.1252/jcej.19.125. ISSN 00219592 
Kong, R., Kim, S.: Characterization of horizontal air-water two-phase flow. Nucl. Eng. Des. 312, 266-276 (2017). https://doi.org/10.1016/j.nucengdes.2016.06.016. ISSN 00295493

Kong, R., Kim, S., Bajorek, S., Tien, K., Hoxie, C.: Effects of pipe size on horizontal two-phase flow: flow regimes, pressure drop, two-phase flow parameters, and drift-flux analysis. Exp. Therm. Fluid Sci. 96(December 2017), 75-89 (2018). https://doi.org/10.1016/j.expthermflusci.2018.02.030. ISSN 08941777

Lockhart, R.W., Martinelli, R.C.: Proposed correlation of data for isothermal two-phase, two-component flow in pipes. Chem. Eng. Prog. 45(1), 39-48 (1949). https://doi.org/10.1016/0017-9310(67)90047-6. ISSN 03607275

Mandhane, J.M., Gregory, G.A., Aziz, K.: A flow pattern map for gas-liquid flow in horizontal pipes. Int. J. Multiph. Flow 1(4), 537-553 (1974). https://doi.org/10.1016/0301-9322(74)90006-8. ISSN 03019322

Mao, F., Desir, F.K., Ebadian, M.A.: Pressure drop measurment and correlation for three-phase flow of simulated nuclear waste in a horizontal pipe. Int. J. Multiph. Flow 23(2), 397-402 (1997)

Miao, Z., Grift, T.E., Hansen, A.C., Ting, K.C.: An overview of lignocellulosic biomass feedstock harvest, processing and supply for biofuel production. Biofuels 4(1), 5-8 (2013). https://doi.org/10.4155/ bfs.12.76. ISSN 1759-7269

Orell, A.: The effect of gas injection on the hydraulic transport of slurries in horizontal pipes. Chem. Eng. Sci. 62(23), 6659-6676 (2007). https://doi.org/10.1016/j.ces.2007.07.067. ISSN 00092509

Rahman, M.A., Adane, K.F., Sanders, R.S.: An improved method for applying the Lockhart-Martinelli correlation to three-phase gas-liquid-solid horizontal pipeline flows. Can. J. Chem. Eng. 91(8), 13721382 (2013). https://doi.org/10.1002/cjce.21843. ISSN 00084034

Rzehak, R., Krepper, E.: CFD modeling of bubble-induced turbulence. Int. J. Multiph. Flow 55, 138-155 (2013). https://doi.org/10.1016/j.ijmultiphaseflow.2013.04.007. ISSN 03019322

Sassi, P., Pallarès, J., Stiriba, Y.: Visualization and measurement of two-phase flows in horizontal pipelines. Exp. Comput. Multiph. Flow 2(1), 41-51 (2020). https://doi.org/10.1007/s42757-019-0022-1

Scott, D.S., Rao, P.K.: Transport of solids by gas-liquid mixtures in horizontal pipes. Can. J. Chem. Eng. 49, 302-309 (1971). ISSN 0722-1819

Spedding, P.L., Spence, D.R.: Flow regimes in two-phase gas-liquid flow. Int. J. Multiph. Flow 19(2), 245280 (1993). https://doi.org/10.1016/0301-9322(93)90002-C. ISSN 0301-9322

Stutz, B., Reghem, P., Martinez, O.: Flow of slurries of particles with density close to that of water. In: 2 nd Workshop on Ice Slurries of IIF/IIR, pp. 1-12 (2000)

Sun, L., Mishima, K.: Evaluation analysis of prediction methods for two-phase flow pressure drop in mini-channels. Int. J. Multiph. Flow 35(1), 47-54 (2009). https://doi.org/10.1016/j.ijmultiphaseflo w.2008.08.003. ISSN 03019322

Taitel, Y., Dukler, A.E.: A model for predicting flow regime transitions in horizontal and near horizontal gas-liquid flow. AIChE J. 22(1), 47-55 (1976). https://doi.org/10.1002/aic.690220105

Talley, J.D., Worosz, T., Kim, S., Buchanan, J.R.: Characterization of horizontal air-water two-phase flow in a round pipe Part I: flow visualization. Int. J. Multiph. Flow 76, 212-222 (2015a). https://doi. org/10.1016/j.ijmultiphaseflow.2015.06.011. ISSN 0301-9322

Talley, J.D., Worosz, T., Kim, S.: Characterization of horizontal air-water two-phase flow in a round pipe Part II: measurement of local two-phase parameters in bubbly flow. Int. J. Multiph. Flow 76, 223-236 (2015b). https://doi.org/10.1016/j.ijmultiphaseflow.2015.06.012. ISSN 03019322

Wilson, K.C., Sellgren, A.: Interaction of particles and near-wall lift in slurry pipelines. J. Hydraul. Eng. 129(January), 73-76 (2003). https://doi.org/10.1061/(ASCE)0733-9429(2003)129:1(73). ISSN 07339429 